\title{
On modules over group rings of groups with restrictions on the system of all proper subgroups
}

\author{
O.Yu.Dashkova \\ Department of Mathematics and Mechanics, Dnepropetrovsk National University, prospekt \\ Gagarina, 72, 49010, Dnepropetrovsk, Ukraine, odashkova@yandex.ru
}

\begin{abstract}
We consider the class $\mathfrak{M}$ of $\mathbf{R}$-modules where $\mathbf{R}$ is an associative ring. Let $A$ be a module over a group ring $\mathbf{R} G$ where $G$ is a group and let $\mathfrak{L}(G)$ be a set of all proper subgroups of $G$ such that if $H \in \mathfrak{L}(G)$ then $A / C_{A}(H)$ belongs to $\mathfrak{M}$. We study an $\mathbf{R} G$-module $A$ such that $G \neq G^{\prime}, C_{G}(A)=1, A / C_{A}(G) \notin \mathfrak{M}$, and $\mathfrak{M}$ is one of the classes: artinian $\mathbf{R}$-modules, minimax $\mathbf{R}$-modules, finite $\mathbf{R}$-modules. We consider the cases: 1) $\mathfrak{M}$ is a class of all artinian $\mathbf{R}$-modules, $\mathbf{R}$ is either a ring of integers or a ring of $p$-adic integers; 2) $\mathfrak{M}$ is a class of all minimax $\mathbf{R}$-modules, $\mathbf{R}$ is a ring of integers, $\mathbf{G}$ is a locally soluble group; 3) $\mathfrak{M}$ is a class of all finite $\mathbf{R}$-modules, $\mathbf{R}$ is an associative ring. In these cases we prove that $G$ is isomorphic to a quasi-cyclic $q$-group for some prime $q$.
\end{abstract}

\section{Keywords: Module over group ring; artinian module; minimax module}

\section{AMS Subject Classification: 20F19, 20H25}

\section{$\S 1$. Introduction}

Investigation of modules over group rings is an important direction in algebra. Modules over group rings of finite groups have been studied by many authors. If $G$ is an infinite group, the situation is totally different. The study of this case requires some additional restrictions. Modules over group rings of infinite groups have been considered recently in [8]. Artinian and noetherian modules over group rings are a broad class of modules over group rings. Recall that a module is called artinian if the partially ordered set of all its submodules satisfies the minimal condition. A module is called noetherian if the partially ordered set of all its submodules satisfies the maximal condition. Natural generalization of the classes of artinian and noetherian modules is the class of minimax modules (chapter 7 [6]). Let $A$ be an $\mathbf{R}$-module, $\mathbf{R}$ be an associative ring. $\mathbf{R}$-module $A$ is called minimax if it has the finite series of submodules such that every its factor is either a noetherian $\mathbf{R}$-module or an artinian $\mathbf{R}$-module. It arises the question on investigation of modules over group rings which are not artinian or noetherian or else minimax but are similar to these modules in some sence.

Let $\mathfrak{M}$ be a class of $\mathbf{R}$-modules where $\mathbf{R}$ is an associative ring and let $A$ be a module over a group $\operatorname{ring} \mathbf{R} G$ where $G$ is a group. Let $\mathfrak{L}(G)$ be a set of subgroups of $G$ such that 
if $H \in \mathfrak{L}(G)$ then $A / C_{A}(H)$ belongs to $\mathfrak{M}$. B.A.F. Wehrfritz have considered groups $G$ of automorphisms of a module $A$ over a ring $\mathbf{R}$ if $\mathfrak{M}$ is one of the classes of noetherian, artinian or finite $\mathbf{R}$-modules and $\mathfrak{L}(G)$ contains all finitely generated subgroups of $G$ [11] [14], [16].

Let $A$ be an $\mathbf{R} G$-module such that all proper subgroups of $G$ belong to $\mathfrak{L}(G)$ but $G$ does not belong to $\mathfrak{L}(G)$. If $A$ is an $\mathbf{R} G$-module, $\mathbf{R}=F$ is a field of prime characteristic, $C_{G}(A)=1, G$ is an almost locally soluble group then $G$ is isomorphic to a quasi-cyclic $q$-group for some prime $q$ [3]. It was investigated the case where $A$ is an $\mathbf{R} G$-module, $\mathbf{R}$ is a ring $\mathbb{Z}_{p \infty}$ of $p$-adic integers, $C_{G}(A)=1, G$ is an infinite soluble group and $\mathfrak{M}$ is a class of all artinian $\mathbf{R}$-modules [2]. It was proved that $G$ is isomorphic to a quasi-cyclic $q$-group for some prime $q$ also. In [1] it was considered the case where $A$ is an $\mathbf{R} G$-module, $\mathbf{R}$ is a ring $\mathbb{Z}$ of integers, $C_{G}(A)=1, G$ is an infinite soluble group and $\mathfrak{M}$ is a class of all artinian $\mathbf{R}$-modules. In this case $G$ is isomorphic to a quasi-cyclic $q$-group for some prime $q$ too.

In [7] the authors investigated the case where $A$ is an $\mathbf{R} G$-module, $\mathbf{R}$ is a ring $\mathbb{Z}$ of integers, $C_{G}(A)=1, G$ is a locally generalized radical group and $\mathfrak{M}$ is a class of all artinianby-(finite rank) $\mathbf{R}$-modules.

We study $\mathbf{R} G$-module $A$ such that $G$ is an infinite group, $G \neq G^{\prime}, C_{G}(A)=1, A / C_{A}(G) \notin$ $\mathfrak{M}$ and $\mathfrak{M}$ is one of the classes: 1) artinian $\mathbf{R}$-modules; 2) minimax $\mathbf{R}$-modules; 3) finite $\mathbf{R}$-modules. It should be noted that the class of groups which are different from their derived subgroups is sufficiently broad. It contains all soluble groups, $Z D$-groups and free groups. The main results of this work are theorems 2.1-2.3.

\section{§2. On modules over group rings of groups}

\section{with restrictions on the system of all proper subgroups}

Later we consider $\mathbf{R} G-$ module $A$ such that $C_{G}(A)=1, \mathbf{R}$ is an associative ring. At first we prove some preliminary results.

Lemma 2.1. Let $A$ be an $\mathbf{R} G$-module, $K, L$ be subgroups of $G$. If $A / C_{A}(K)$ and $A / C_{A}(L)$ are minimax $\mathbf{R}$-modules then $A / C_{A}(\langle K, L\rangle)$ is a minimax $\mathbf{R}$-module also.

Lemma 2.2. Let $A$ be an $\mathbf{R} G$-module where $G$ is an infinite group, $G \neq G^{\prime}, \mathbf{R}$ is an associative ring. If $A / C_{A}(G)$ is not a minimax $\mathbf{R}$-module and $A / C_{A}(H)$ is a minimax $\mathbf{R}$-module for every proper subgroup $H$ of $G$ then $G$ has not proper subgroups of finite index and $G / G^{\prime}$ is isomorphic to a quasi-cyclic $q$-group for some prime $q$.

Proof. We prove that $G$ is an infinite generated group. Otherwise let $\left\{x_{1}, x_{2}, \cdots, x_{m}\right\}$ be a minimal system of generatings of $G$. If $m=1$ then $G$ is an infinite cyclic group. Therefore $G$ is generated by two proper subgroups. By lemma $2.1 A / C_{A}(G)$ is a minimax $\mathbf{R}$-module. Contradiction. If $k>1$ then $G$ is generated by proper subgroups $\left\langle x_{1}, x_{2}, \cdots, x_{m-1}\right\rangle$ and $\left\langle x_{m}\right\rangle$. We have a contradiction also. It follows that $G$ is an infinite generated group. Now we prove that $G$ has not proper subgroups of finite index. Otherwise if $N$ is a proper subgroup of $G$ of finite index then we can choose a finitely generated subgroup $M$ such that $G=M N$ where $M$ and $N$ are proper subgroups of $G$. By lemma $2.1 A / C_{A}(G)$ is a minimax $\mathbf{R}$-module. Contradiction.

Let $D$ be a derived subgroup of $G$. As $G$ has not proper subgroups of finite index then $G / D$ is infinite. By lemma 2.1 an abelian quotient group $G / D$ can not be generated by two proper subgroups. Let $G / D$ be a nonperiodic group and $T / D$ be a periodic part of $G / D$. Then $G / T$ is generated by two proper subgroups. Contradiction with lemma 2.1. Therefore $G / D$ is periodic. Hence $G / D$ is isomorphic to a quasi-cyclic $q$-group for some prime $q$ (p.152 [5]). The lemma is proved. 
Lemmas 2.1 and 2.2 are valid if the minimax condition is replaced by the artinian condition or the finiteness condition.

Lemma 2.3. Let $A$ be an artinian $\mathbf{R}$-module where $\mathbf{R}=\mathbb{Z}_{p^{\infty}}$ is a ring of $p$-adic integers. Then the additive group of $A$ is Chernikov and its divisible part is a $p$-group.

Proof. Let $P$ be a maximal ideal of $\mathbf{R}$. Then the additive group of $\mathbf{R} / P$ has an order $p$ and the additive group of $\mathbf{R} / P^{k}$ is a cyclic group of an orger $p^{k}$. Let $\mathbf{R} / P^{k}=\left\langle a_{k}\right\rangle$, $k=1,2, \cdots, \pi_{k}^{k+1}: \mathbf{R} / P^{k} \longrightarrow \mathbf{R} / P^{k+1}$ where

$$
\pi_{k}^{k+1}\left(a_{k}\right)=p a_{k+1}
$$

and let $\pi_{k}^{m}: \mathbf{R} / P^{k} \longrightarrow \mathbf{R} / P^{m}$ where $m>k$ and

$$
\pi_{k}^{m}\left(a_{k}\right)=p^{m-k} a_{m}
$$

We consider an injective limit of the set of $\mathbf{R} / P^{k}, k=1,2, \cdots, n, \cdots$. From the choice of $a_{1}$ it follows that $p a_{1}=0$. Therefore this injective limit is isomorphic to a quasi-cyclic $p$-group $C_{p^{\infty}}$. It follows that an additive group of a Prüfer $\mathbf{R}$-module is isomorphic to a quasicyclic $p$-group $C_{p^{\infty}}$ (ch. 5 [6]). By theorem 7.13 [6] an artinian $\mathbf{R}$-module is decomposed in a direct sum $A=a_{1} \mathbf{R} \oplus a_{2} \mathbf{R} \oplus \cdots \oplus a_{n} \mathbf{R} \oplus C_{1} \oplus \cdots \oplus C_{k}$ where $C_{i}$ is a Prüfer $P_{i}$-module, $P_{i} \in \operatorname{Spec}(\mathbf{R}), i=1, \cdots, k, \operatorname{Ann}_{\mathbf{R}}\left(a_{j}\right)=P_{j}^{m_{j}}, P_{j} \in \operatorname{Spec}(\mathbf{R}), j=1, \cdots, n$. Every ideal of $\mathbf{R}$ has a finite index in $\mathbf{R}$ (ch. 6 [5]). Therefore $a_{j} \mathbf{R}$ is a finite $\mathbf{R}$-module for each $j=1, \cdots, n$. It follows that the additive group of an artinian $\mathbb{Z}_{p^{\infty}}$-module $A$ is Chernikov and its divisible part is a $p$-group. The lemma is proved.

Now we prove the main results of this work.

Theorem 2.1. Let $A$ be an $\mathbf{R} G$-module where $G$ is an infinite group, $G \neq G^{\prime}, \mathbf{R}$ is either a ring $\mathbb{Z}$ of integers or a ring $\mathbb{Z}_{p^{\infty}}$ of $p$-adic integers. If $A / C_{A}(G)$ is not an artinian $\mathbf{R}$-module and $A / C_{A}(H)$ is an artinian $\mathbf{R}$-module for every proper subgroup $H$ of $G$ then $G$ is isomorphic to a quasi-cyclic $q$-group $C_{q^{\infty}}$ for some prime $q$.

Proof. Let $D$ be a derived subgroup of $G$. By lemma $2.2 G / D$ is isomorphic to a quasicyclic $q$-group for some prime $q$. Let $H / D$ be any finite subgroup of $G / D$. Since $H$ is a proper subgroup of $G$ then $A / C_{A}(H)$ is an artinian $\mathbf{R}$-module. If $\mathbf{R}$ is a ring $\mathbb{Z}$ of integers then $A / C_{A}(H)$ is an abelian group with the minimal condition for subgroups. Therefore $A / C_{A}(H)$ is a Chernikov group. If $\mathbf{R}$ is a ring $\mathbb{Z}_{p^{\infty}}$ of $p$-adic integers then $A / C_{A}(H)$ is a Chernikov group by lemma 2.3. It follows that $A / C_{A}(H)$ is the union of finite characteristic subgroups $A_{n} / C_{A}(H), n=1,2, \cdots$, and for each $n=1,2, \cdots$, we have that $G / C_{G}\left(A_{n} / C_{A}(H)\right)$ is finite. By lemma $2.2 G$ has not proper subgroups of finite index. Then $G=C_{G}\left(A_{n} / C_{A}(H)\right)$ for each $n=1,2, \cdots$. It follows that $\left[G, A_{n}\right] \leq C_{A}(H)$ for each $n=1,2, \cdots$. Therefore $[G, A] \leq C_{A}(H)$. From the choice of $H$ it follows that $[G, A] \leq C_{A}(G)$ and so $G$ acts trivially at every factor of the series $0 \leq C_{A}(G) \leq A$. By Kaluzhnin theorem (p. 144 [4]) $G$ is an abelian group. It follows that $G$ is isomorphic to a quasi-cyclic $q$-group $C_{q^{\infty}}$ for some prime $q$. The theorem is proved.

Theorem 2.2. Let $A$ be a $\mathbb{Z} G$-module where $G$ is an infinite locally soluble group, $G \neq G^{\prime}, \mathbb{Z}$ is a ring of integers. If $A / C_{A}(G)$ is not a minimax $\mathbb{Z}$-module and $A / C_{A}(H)$ is a minimax $\mathbb{Z}$-module for every proper subgroup $H$ of $G$ then $G$ is isomorphic to a quasi-cyclic $q$-group $C_{q^{\infty}}$ for some prime $q$.

Proof. Let $D$ be a derived subgroup of $G$. By lemma $2.2 G / D$ is isomorphic to a quasicyclic $q$-group for some prime $q$. At first we consider the case where there is the proper subgroup $L$ such that $D \leq L$ and $A / C_{A}(L)$ is not an artinian $\mathbb{Z}$-module. In this case for any proper subgroup $H$ such that $L \leq H$ it is existed a series of $\mathbb{Z} G$-submodules

$$
0 \leq C_{A}(H) \leq A_{1} \leq A
$$


such that the additive group of $A_{1} / C_{A}(H)$ is either an abelian Chernikov group or trivial, the additive group of $A / A_{1}$ is an abelian torsion-free group of finite 0 -rank. Therefore we can construct the series of $\mathbb{Z} G$-submodules

$$
0 \leq C_{A}(H) \leq A_{1} \leq A_{2} \leq \cdots \leq A_{n-1} \leq A_{n}=A
$$

such that the additive group of $A_{1} / C_{A}(H)$ is either abelian Chernikov or trivial, $A_{k+1} / A_{k}$, $k=1, \cdots, n-1$, are $G$-rationally irreducible and the additive groups of $A_{k+1} / A_{k}$ are abelian torsion-free groups of finite 0 -rank.

Now we consider the series of $\mathbb{Z} G$-submodules

$$
\begin{gathered}
\overline{0}=C_{A}(H) / C_{A}(H) \leq A_{1} / C_{A}(H) \leq A_{2} / C_{A}(H) \leq \cdots \\
\leq A_{n-1} / C_{A}(H) \leq A_{n} / C_{A}(H)=A / C_{A}(H) .
\end{gathered}
$$

If the additive group of $A_{1} / C_{A}(H)$ is non-trivial then $A_{1} / C_{A}(H)$ is the union of finite characteristic subgroups $B_{m} / C_{A}(H), m=1,2, \cdots$, and for each $m=1,2, \cdots$, we have that $G / C_{G}\left(B_{m} / C_{A}(H)\right)$ is finite. By lemma $2.2 G$ has not proper subgroups of finite index. Then $G=C_{G}\left(B_{m} / C_{A}(H)\right)$ for each $m=1,2, \cdots$. Therefore $\left[G, B_{m}\right] \leq C_{A}(H)$ for each $m=1,2, \cdots$. It follows that $\left[G, A_{1}\right] \leq C_{A}(H)$. So $G$ acts trivially at the factor $A_{1} / C_{A}(H)$. For any $k=1, \cdots, n-1$, we have the isomorphism of $\mathbb{Z} G$-modules

$$
\left(A_{k+1} / C_{A}(H)\right) /\left(A_{k} / C_{A}(H)\right) \simeq A_{k+1} / A_{k} .
$$

The quotient group $G / C_{G}\left(A_{k+1} / A_{k}\right), k=1, \cdots, n-1$, can be considered as an irreducible subgroup of $G L_{r}(\mathbb{Q})$. By corollary $3.8[15] G / C_{G}\left(A_{k+1} / A_{k}\right)$ is a soluble group for each $k=1, \cdots, n-1$. By A.I.Maltzev theorem (lemma 3.5 [15]) $G / C_{G}\left(A_{k+1} / A_{k}\right), k=1, \cdots, n-1$, are almost abelian. Since $G$ has not proper subgroups of finite index then $G / C_{G}\left(A_{k+1} / A_{k}\right)$, $k=1, \cdots, n-1$, are abelian. From $D \leq C_{G}\left(A_{k+1} / A_{k}\right)$ it follows that for any $k=1, \cdots, n-1$, the quotient group $G / C_{G}\left(A_{k+1} / A_{k}\right)$ is either isomorphic to a quasi-cyclic $q$-group for some prime $q$ or trivial. For each $k=1, \cdots, n-1$, the additive group of $A_{k+1} / A_{k}$ is an abelian $A_{4}$-group [9]. Since a periodic subgroup of automorphisms group of a soluble $A_{4}$-group is finite [10], then $G / C_{G}\left(A_{k+1} / A_{k}\right), k=1, \cdots, n-1$, are trivial. Therefore $G$ acts trivially at every factor of the series

$$
\overline{0} \leq A_{1} / C_{A}(H) \leq A_{2} / C_{A}(H) \leq \cdots \leq A_{n-1} / C_{A}(H) \leq A_{n} / C_{A}(H)=A / C_{A}(H) .
$$

At first we consider the case where there exists a subgroup $M$ such that $D \leq M, M / D$ is a finite subgroup of $G / D, A / C_{A}(M)$ is not an artinian $\mathbb{Z}$-module and for any proper subgroup $H$ of $G$ such that $M \leq H$ the equalities $A_{1}=C_{A}(H), A_{2}=A$ are valid. As we proved $G$ acts trivially at the factor $A_{2} / A_{1}=A / C_{A}(H)$. Therefore $[G, A] \leq C_{A}(H)$. From the choice of $H$ it follows that $[G, A] \leq C_{A}(G)$. So $G$ acts trivially at every factor of the series $0 \leq C_{A}(G) \leq A$. It follows that $G$ is an abelian group. Therefore $G$ is isomorphic to a quasi-cyclic $q$-group. Otherwise we can choose a subgroup $H$ such that $H / D$ is a finite subgroup of $G / D, A / C_{A}(H)$ is not an artinian $\mathbb{Z}$-module and even if one from the equalities $A_{1}=C_{A}(H), A_{2}=A$ is not valid. Then by Kaluzhnin theorem (p. 144 [4]) $G$ is a nilpotent group of step $\leq n-1$. Let

$$
\langle 1\rangle=Z_{0} \leq Z_{1} \leq Z_{2} \leq \cdots \leq Z_{l-1} \leq Z_{l}=G
$$

be an upper central series of $G$, where $l \leq n-1$. From $D \leq Z_{l-1}$ it follows that $G / Z_{l-1}$ is isomorphic to a quasi-cyclic $q$-group for some prime $q$. Therefore $G / Z_{l-2}$ is an extension 
of a central subgroup by a quasi-cyclic $q$-group. Then $G / Z_{l-2}$ is abelian. By lemma 2.2 $G / Z_{l-2}$ is isomorphic to a quasi-cyclic $q$-group for some prime $q$. If we continue similarly at the step with the number $l-1$ we obtain that $G / Z_{1}$ is isomorphic to a quasi-cyclic $q$-group for some prime $q$. It follows that $G$ is an extension of a central subgroup by a quasi-cyclic $q$-group. Therefore $G$ is an abelian group and $G$ is isomorphic to a quasi-cyclic $q$-group $C_{q^{\infty}}$ for some prime $q$.

Now we consider the case where for every proper subgroup $H$ such that $D \leq H$ the quotient module $A / C_{A}(H)$ is an artinian $\mathbb{Z}$-module. It follows from theorem 3.1 [1] that $G$ is isomorphic to a quasi-cyclic $q$-group $C_{q^{\infty}}$ for some prime $q$. The theorem is proved.

It should be noted that theorem 2.2 is the special case of the main theorem of [7].

Theorem 2.3. Let $A$ be an $\mathbf{R} G$-module where $G$ is an infinite group, $G \neq G^{\prime}, \mathbf{R}$ is an associative ring. If $A / C_{A}(G)$ is an infinite $\mathbf{R}$-module and $A / C_{A}(H)$ is a finite $\mathbf{R}$-module for every proper subgroup $H$ of $G$ then $G$ is isomorphic to a quasi-cyclic $q$-group $C_{q^{\infty}}$ for some prime $q$.

Proof. Let $D$ be a derived subgroup of $G$. By lemma $2.2 G / D$ is isomorphic to a quasicyclic $q$-group for some prime $q$. Let $H / D$ be any finite subgroup of $G / D$. Since $H$ is a proper subgroup of $G$ then $A / C_{A}(H)$ is finite. Therefore $G / C_{G}\left(A / C_{A}(H)\right)$ is finite. As by lemma $2.2 G$ has not proper subgroups of finite index then $G=C_{G}\left(A / C_{A}(H)\right)$. It follows that $[G, A] \leq C_{A}(H)$. From the choice of $H$ it follows that $[G, A] \leq C_{A}(G)$. So $G$ acts trivially at every factor of the series $0 \leq C_{A}(G) \leq A$. By Kaluzhnin theorem (p. 144 [4]) $G$ is an abelian group. Therefore $G$ is isomorphic to a quasi-cyclic $q$-group $C_{q^{\infty}}$ for some prime $q$. The theorem is proved.

In [7] the authors have constructed the example of a module with the prescribed conditions.

\section{References}

[1] O.Yu. Dashkova, Locally soluble AFA-groups, Ukr. Math. J. 65 (2013). P. 459-469.

[2] O.Yu. Dashkova, On modules over group rings of locally soluble groups for a ring of p-adic integers, Algebra Discrete Math. no. 1 (2009). P. 32-43.

[3] M.R. Dixon M.R., M.J. Evans, L.A. Kurdachenko, Linear groups with the minimal condition on subgroups of infinite central dimension, J. Algebra. 277 (2004). P. 172-186.

[4] M.I. Kargapolov, Yu.I. Merzlyakov, Bases of the Theory of Groups (Nauka: Moskow, 1975).

[5] A.G. Kurosh, The Theory of Groups (Nauka: Moskow, 1967).

[6] L.A. Kurdachenko, I.Ya. Subbotin, N.N. Semko, Insight into Modules over Dedekind Domains (National Academy of Sciences of Ukraine. Institute of Mathematics, Kiev, 2008).

[7] L.A. Kurdachenko, I.Ya. Subbotin, V.A. Chepurdya, On the structure of some modules over generalized soluble groups, arXiv:1302.2115.

[8] L.A. Kurdachenko, J. Otal, I.Ya. Subbotin, Artinian Modules over Group Rings (Birkhäuser, Boston, Berlin, 2007).

[9] A.I. Maltzev, On some classes of infinite soluble groups, Mat. Sb. 28 (1951). P. 567-588.

[10] D.M. Smirnov, On automorphisms of soluble groups, Dokl. Math. 84 (1952). P. 891-894. 
[11] B.A.F. Wehrfritz, Artinian-finitary groups are locally normal-finitary, J. Algebra. 287 (2005). P. 417-431.

[12] B.A.F. Wehrfritz, Artinian-finitary groups over commutative rings, Illinois J. Math. 47 (2003). P. 551-565.

[13] B.A.F. Wehrfritz, Artinian-finitary groups over commutative rings and noncommutative rings, J. Lond. Math. Soc. (2). 70 (2004). P. 325-340.

[14] B.A.F. Wehrfritz, Finite-finitary groups of automorphisms, J. Algebra Appl. 1 (2002). P. 375-389.

[15] B.A.F. Wehrfritz, Infinite Linear Groups ( Ergebnisse der Mathematik und ihrer Grenzgebiete, Springer-Verlag, New York, Heidelberg, Berlin, 1973).

[16] B.A.F. Wehrfritz, On generalized finitary groups, J. Algebra. 247 (2002). P. 707-727. 